PROCEEDINGS OF THE

AMERICAN MATHEMATICAL SOCIETY

Volume 130, Number 1, Pages 303-306

S 0002-9939(01)06384-

Article electronically published on July 31, 2001

\title{
SMOOTH DIAMETER AND EIGENVALUE RIGIDITY IN POSITIVE RICCI CURVATURE
}

\author{
WILDERICH TUSCHMANN
}

(Communicated by Wolfgang Ziller)

\begin{abstract}
A recent injectivity radius estimate and previous sphere theorems yield the following smooth diameter sphere theorem for manifolds of positive Ricci curvature: For any given $m$ and $C$ there exists a positive constant $\varepsilon=$ $\varepsilon(m, C)>0$ such that any $m$-dimensional complete Riemannian manifold with Ricci curvature Ricc $\geq m-1$, sectional curvature $K \leq C$ and diameter $\geq \pi-\varepsilon$ is Lipschitz close and diffeomorphic to the standard unit $m$-sphere. A similar statement holds when the diameter is replaced by the first eigenvalue of the Laplacian.
\end{abstract}

Let $M$ be a complete Riemannian manifold of dimension $m \geq 2$ with Ricci curvature Ricc $\geq m-1$. Then, by the Bonnet-Myers theorem, the diameter of $M$ is bounded from above by $\pi$, and by Cheng's maximal diameter sphere theorem the maximal value $\pi$ is attained if and only if $M$ is isometric to the unit $m$-sphere (see Chg).

In contrast to the sectional curvature setting, where Grove and Shiohama (see GrS]) showed that a complete Riemannian $m$-manifold with sectional curvature $\geq 1$ and diameter $>\pi / 2$ must be homeomorphic to the $m$-dimensional sphere, and where until today no example of an exotic sphere with positive sectional curvature is known, in the case of positive Ricci curvature however the following phenomena arise: First of all, Anderson and Otsu (see [An1], Ot1] ) showed that Cheng's result is not even topologically rigid by constructing, for $m \geq 4$ on closed smooth $m$ manifolds whose homotopy type is distinct from that of the sphere, Riemannian metrics with $R i c c \geq m-1$ and diameter arbitrarily close to $\pi$. Secondly, it is known that many exotic spheres, i.e., smooth manifolds which are homeomorphic, but not diffeomorphic to a standard sphere, carry metrics of positive Ricci curvature

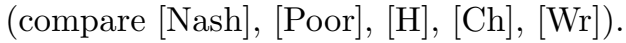

Thus additional assumptions are needed to obtain sphere theorems for positively Ricci curved manifolds, and of particular interest here is the problem of finding conditions which guarantee smooth instead of merely topological rigidity. Contributions to this field of questions have been made in particular by Anderson, Bessa, Brittain, Cai, Colding, Eschenburg, Grove-Petersen, Itokawa, Katsuda, Nakamura, Otsu, Paeng, Perelman, Petersen, Petersen-Zhu, Shiohama, Wilhelm, Wu, Xia, and

Received by the editors October 26, 2000.

2000 Mathematics Subject Classification. Primary 53C20.

Key words and phrases. Sphere theorems, injectivity radius, exotic spheres, positive Ricci curvature. 


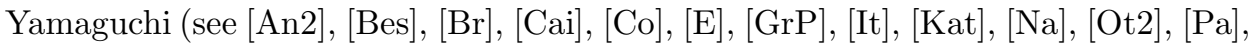
Per, [Pet, PZ, Shi1, Wi], [Wu, Xia, Yam]; compare also the survey Shi2]).

Except for Perelman's topological diameter sphere theorem (see [Per]), stating that for any given $m$ and $C$ there exists a positive constant $\varepsilon=\varepsilon(m, C)>0$ such that any $m$-dimensional complete Riemannian manifold with Ricci curvature Ricc $\geq m-1$, sectional curvature $K \geq C$ and diameter $\geq \pi-\varepsilon$ is a twisted sphere, all of these results use further assumptions on additional geometric quantities besides various conditions on curvature and diameter. The purpose of this note is to point out that by combining Perelman's result and previous smooth sphere theorems with a recent injectivity radius estimate one obtains, along with other consequences, the following differentiable sphere theorem which requires only assumptions on curvature and diameter:

1.1 Theorem. For any given $m$ and $C$ there exists a positive constant $\varepsilon(m, C)>0$ such that for $\varepsilon \leq \varepsilon(m, C)$ any $m$-dimensional complete Riemannian manifold $M$ with Ricci curvature Ricc $\geq m-1$, sectional curvature $K \leq C$ and diameter $\geq \pi-\varepsilon$ is diffeomorphic to the standard $m$-sphere. Moreover, the Lipschitz distance between $M$ and the unit $m$-sphere $S^{m}(1)$ converges to zero as $\varepsilon \rightarrow 0$.

Lichnerowicz (see [Li]) showed that the first eigenvalue $\lambda_{1}(M)$ of the Laplacian of a complete Riemannian $m$-manifold with Ricci curvature Ricc $\geq m-1$ is bounded from below by $m$, and by Obata's minimal eigenvalue sphere theorem (see $\mathrm{Ob}$ ) $\lambda_{1}(M)$ is equal to $m$ if and only if $M$ is isometric to the unit $m$-sphere. It follows from results of Croke and Cheng (see $[\mathrm{Cr}], \overline{\mathrm{Chg}} \mid$ ) that Theorem 1.1 is equivalent to the following smooth eigenvalue pinching sphere theorem:

1.2 Theorem. For any given $m$ and $C$ there exists a positive constant $\varepsilon^{\prime}(m, C)>0$ such that for $\varepsilon \leq \varepsilon^{\prime}(m, C)$ any $m$-dimensional complete Riemannian manifold $M$ with Ricci curvature Ricc $\geq m-1$, sectional curvature $K \leq C$ and $\lambda_{1}(M) \leq m+\varepsilon$ is diffeomorphic to the standard $m$-sphere. Moreover, the Lipschitz distance between $M$ and the unit $m$-sphere $S^{m}(1)$ converges to zero as $\varepsilon \rightarrow 0$.

Theorems 1.1 and 1.2 and sphere theorems as in Bes imply that a violation of smooth rigidity in Cheng's maximal diameter or Obata's minimal first eigenvalue sphere theorem can only be achieved by sequences of manifolds whose sectional curvatures blow up and whose injectivity radii converge to zero:

1.3 Corollary. Let $\left(M_{n}\right)_{n \in \mathbb{N}}$ be a sequence of complete Riemannian m-manifolds with Ricci curvature Ricc $\geq m-1$ whose diameters converge to $\pi$ or whose first eigenvalues converge to $m$ as $n$ goes to infinity.

Then either all but finitely many $M_{n}$ are diffeomorphic to the standard $m$-sphere, or else $\limsup \sup _{n \rightarrow \infty} K_{M_{n}}=+\infty$ and $\lim _{n \rightarrow \infty} \operatorname{inj}_{M_{n}}=0$.

Theorem 1.1 follows from previous sphere theorems and the following injectivity radius estimate:

1.4 Theorem $([\mathrm{PT}])$. For each $m$ and any $0<\delta \leq 1$ there exists a positive constant $i_{0}(m, \delta)>0$ such that the injectivity radius $i_{g}$ of any Riemannian metric $g$ with Ricci curvature Ricc $\geq(m-1) \delta$ and sectional curvature $K \leq 1$ on a simply connected closed $m$-dimensional manifold with finite second homotopy group is bounded from below by $i_{g} \geq i_{0}(m, \delta)$.

In fact, to prove Theorem 1.1 one observes that since an upper sectional and a lower Ricci curvature bound imply a lower bound for the sectional curvature, 
Perelman's topological diameter sphere theorem can be invoked and one may assume that the manifolds $M$ in question in Theorem 1.1 are twisted spheres, so that in particular Theorem 1.4 applies to them. With this uniform injectivity radius estimate at hand known differentiable sphere theorems, for example the ones of $\overline{\mathrm{Br}}$, $\mathrm{Kat}, \mathrm{Bes}, \mathrm{Pa}$, or $[\mathrm{Yam}$, now easily yield the theorem's conclusions.

The question remains whether the upper sectional curvature bound in Theorem 1.1 is indeed indispensable, or whether instead the assumptions in Perelman's theorem might already imply diffeomorphism to $S^{m}$. To construct Riemannian metrics on exotic spheres which violate smooth rigidity seems in view of Corollary 1.3 actually rather difficult.

It is a pleasure to thank the referee for constructive remarks.

\section{REFERENCES}

[An1] M. T. Anderson, Metrics of positive Ricci curvature with large diameters, Man. Math. 68 (1990) 405-415 MR 91g:53045

[An2] M. T. Anderson, Convergence and rigidity of manifolds under Ricci curvature bounds, Inv. Math. 102 (1990) 429-445 MR 92c:53024

[Br] D. Brittain, A Diameter Pinching Sphere Theorem for Positive Ricci Curvature, Preprint (seemingly unpublished, compare however the result's of the proof in [Gr])

[Bes] G. Bessa, Differentiable sphere theorems for Ricci curvature, Math. Z. 214 (1993) 245-249 MR 94g:53035

[Cai] M. Cai, Rigidity of manifolds with large volume, Math. Z. 213, No.1 (1993) 17-31 MR 94b:53071

[Ch] J. Cheeger, Some examples of manifolds of non-negative curvature, J. Diff. Geom. 8 (1973) 623-628 MR 49:6085

[Chg] Y. Cheng, Eigenvalue comparison theorems and its geometric applications, Math. Z. 143 (1975) 289-297 MR 51:14170

[Co] T. Colding, Large manifolds with positive Ricci curvature, Invent. Math. 124 (1996) 193214 MR 96k:53068

[Cr] C. B. Croke, An eigenvalue pinching theorem, Invent. Math. 68 (1982) 253-256 MR 84a:58084

[E] J.-H. Eschenburg, Diameter, volume, and topology for positive Ricci curvature, J. Diff. Geom. 33 (1991) 743-747 MR 92b:53054

[Gr] K. Grove, Metric differential geometry, Differential geometry (Lyngby, 1985), 171-227, Lecture Notes in Math., 1263, Springer, Berlin-New York, 1987 MR 88i:53075

[GrP] K. Grove and P. Petersen, A pinching theorem for homotopy spheres, J.A.M.S. 3,3 (1990) 671-677 MR 91e:53040

[GrS] K. Grove and K. Shiohama, A generalized sphere theorem, Ann. Math. 106 (1977) 201-211 MR 58:18268

[H] H. Hernández-Andrade, A class of compact manifolds with positive Ricci curvature, Differential Geometry, Proc. Symp. Pure Math. 28 (1975) 73-87 MR 52:1565

[It] Y. Itokawa, The topology of certain Riemannian manifolds with positive Ricci curvature, J. Diff. Geom. 18 (1983) 151-155 MR 84i:53044

[Kat] A. Katsuda, Gromov's convergence theorem and its applications, Nagoya Math. J. 100 (1985) 11-48; Erratum: Nagoya Math. J. 114 (1989) 173-174 MR 87e:53067, MR 90e:53057

[Li] A. Lichnerowicz, Géométrie des groupes de transformations, Dunod, Paris (1958) MR 23:A1329

[Na] G. Nakamura, Diameter sphere theorems for manifolds of positive Ricci curvature, Dissertation, Nagoya University 1989

[Nash] J. C. Nash, Positive Ricci curvature on fiber bundles, J. Diff. Geom. 14 (1979) 241-265 MR 81k:53039

[Ob] M. Obata, Certain conditions for a Riemannian manifold to be isometric to a sphere, J. Math. Soc. Japan 14 (1962) 333-340 MR 25:5479

[Ot1] Y. Otsu, On manifolds of positive Ricci curvature with large diameter, Math. Z. 206 (1991) 255-264 MR 91m:53033 
[Ot2] Y. Otsu, On manifolds of small excess, Amer. J. Math. 115 (1993) 1229-1280 MR 95i:53046

[Pa] S.-H. Paeng, A sphere theorem under a curvature perturbation. I; II, Kyushu J. Math. 50 (1996) 459-470; Kyushu J. Math. 52 (1998) 439-454 MR 97m:53068 MR 99k:53081

[Per] G. Perelman, A diameter sphere theorem for manifolds of positive Ricci curvature, Math. Z. 218 (1995) 595-596 MR 96f:53056

[Pet] P. Petersen, Small excess and Ricci curvature, J. Geom. Anal. 1,4 (1991) 383-387 MR 93a:53031

[Poor] W. A. Poor, Some exotic spheres with positive Ricci curvature, Math. Annalen 216 (1975) 245-252 MR 53:3945

[PT] A. Petrunin and W. Tuschmann, Diffeomorphism Finiteness, Positive Pinching, and Second Homotopy, Geom. and Funct. Anal. (GAFA) 9 (1999) 736-774 MR 2000k:53031

[PZ] P. Petersen and S. Zhu, An excess sphere theorem, Ann. Sci. Ec. Norm. Super., IV. Ser. 26, No.2 (1993) 175-188 MR 94b:53077

[Shi1] K. Shiohama, A sphere theorem for manifolds of positive Ricci curvature, Trans. Amer. Math. Soc. 275 (1983) 811-819 MR 84c:53041

[Shi2] K. Shiohama, Sphere Theorems, F.J.E. Dillen and L.C.A. Verstraelen (edts.), Handbook of Differential Geometry, Vol. I, Elsevier Science B.V., Amsterdam 2000, 865-903 MR 2001c:53051

[Wi] F. Wilhelm, On radius, systole, and positive Ricci curvature, Math. Z. 218 (1995) 597-602 MR 96d:53040

[Wr] D. Wraith, Exotic spheres with positive Ricci curvature, J. Diff. Geom. 46 (1997) 638-649 MR 98i: 53058

[Wu] J.-Y. Wu, A diameter pinching sphere theorem for positive Ricci curvature, Proc. A.M.S. 107,3 (1989) 797-802 MR 90h:53045

[Xia] C. Xia, Rigidity and sphere theorem for manifolds with positive Ricci curvature, Manuscr. Math. 85, No.1 (1994) 79-87 MR 95j:53057

[Yam] T. Yamaguchi, Lipschitz convergence of manifolds of positive Ricci curvature with large volume, Math. Ann. 284 (1989) 423-436 MR 90c:53114

Max-Planck-Institute for Mathematics in the Sciences, Inselstrasse, D-04103 Leipzig, GERMANY

E-mail address: tusch@mis.mpg.de 